\title{
Clinical nodal staging scores for prostate cancer: a proposal for preoperative risk assessment
}

\author{
L A Kluth ${ }^{1,2,18}$, F Abdollah ${ }^{3,18}$, E Xylinas ${ }^{1,4}$, M Rieken $^{1,5}$, H Fajkovic $^{6}$, C Seitz $^{6}$, M Sun $^{7}$, P I Karakiewicz ${ }^{7}$,
}

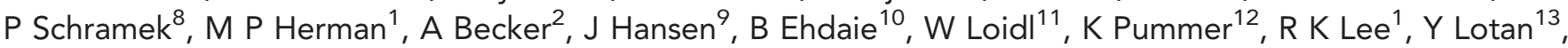
D S Scherr ${ }^{1}$, D Seiler ${ }^{14}$, S A Ahyai ${ }^{2}$, FK-H Chun ${ }^{2}$, M Graefen ${ }^{9}$, A Tewari ${ }^{1}$, A Nonis ${ }^{15}$, A Bachmann ${ }^{5}$, F Montorsi ${ }^{3}$, M Gönen ${ }^{16,19}$, A Briganti ${ }^{3,19}$ and S F Shariat ${ }^{\star 1,1,6,17,19}$

${ }^{1}$ Department of Urology, Weill Medical College of Cornell University, New York Presbyterian Hospital, New York, NY, USA; ${ }^{2}$ Department of Urology, University Medical-Center Hamburg-Eppendorf, Hamburg, Germany; ${ }^{3}$ Department of Urology, Vita-Salute San Raffaele University, Milan, Italy; ${ }^{4}$ Department of Urology, Cochin Hospital, Assistance Publique-Hopitaux de Paris, Paris Descartes University, Paris, France; ${ }^{5}$ Department of Urology, University Hospital of Basel, Basel, Switzerland; ${ }^{6}$ Department of Urology, Medical University of Vienna, Vienna, Austria; ${ }^{7}$ Prognostics and Health Outcomes Unit, University of Montreal Health Center, Montreal, QC, Canada; ${ }^{8}$ Department of Urology, Krankenhaus der Barmherzigen Brueder, Vienna, Austria; ${ }^{9}$ Prostate Cancer Center, Martini-Clinic, Hamburg, Germany; ${ }^{10}$ Department of Urology, Memorial Sloan-Kettering Cancer Center, New York, NY, USA; ${ }^{11}$ Prostate Cancer Center, Krankenhaus Barmherzige Schwestern Linz, Linz, Austria; ${ }^{12}$ Department of Urology, Medical University of Graz, Graz, Austria; ${ }^{13}$ Department of Urology, University of Texas Southwestern Medical Center, Dallas, TX, USA; ${ }^{14}$ Department of Urology, Kantonsspital Aarau, Aarau, Switzerland; ${ }^{15}$ CUSSB (University Centre for Statistics in the Biomedical Sciences), Vita-Salute San Raffaele University, Milan, Italy; ${ }^{16}$ Department of Epidemiology and Biostatistics, Memorial Sloan-Kettering Cancer Center, New York, NY, USA and ${ }^{17}$ Division of Medical Oncology, Weill Medical College of Cornell University, New York Presbyterian Hospital, New York, NY, USA

Background: Pelvic lymph node dissection in patients undergoing radical prostatectomy for clinically localised prostate cancer is not without morbidity and its therapeutical benefit is still a matter of debate. The objective of this study was to develop a model that allows preoperative determination of the minimum number of lymph nodes needed to be removed at radical prostatectomy to ensure true nodal status.

Methods: We analysed data from 4770 patients treated with radical prostatectomy and pelvic lymph node dissection between 2000 and 2011 from eight academic centres. For external validation of our model, we used data from a cohort of 3595 patients who underwent an anatomically defined extended pelvic lymph node dissection. We estimated the sensitivity of pathological nodal staging using a beta-binomial model and developed a novel clinical (preoperative) nodal staging score (cNSS), which represents the probability that a patient has lymph node metastasis as a function of the number of examined nodes.

Results: In the development and validation cohorts, the probability of missing a positive lymph node decreases with increase in the number of nodes examined. A 90\% cNSS can be achieved in the development and validation cohorts by examining 1-6 nodes in cT1 and 6-8 nodes in cT2 tumours. With 11 nodes examined, patients in the development and validation cohorts achieved a cNSS of 90\% and $80 \%$ with cT3 tumours, respectively.

Conclusions: Pelvic lymph node dissection is the only reliable technique to ensure accurate nodal staging in patients treated with radical prostatectomy for clinically localised prostate cancer. The minimum number of examined lymph nodes needed for accurate nodal staging may be predictable, being strongly dependent on prostate cancer characteristics at diagnosis.

\footnotetext{
${ }^{*}$ Correspondence: Professor SF Shariat; E-mail: sfshariat@gmail.com

${ }^{18}$ These authors contributed equally to this work.

${ }^{19}$ These are senior authors.
}

Received 14 January 2014; revised 5 May 2014; accepted 12 May 2014; published online 8 July 2014

(c) 2014 Cancer Research UK. All rights reserved 0007-0920/14 
Lymph node (LN) metastasis is the strongest prognostic factor for patients treated with radical prostatectomy (RP) for clinically localised prostate cancer (PCa) (Briganti et al, 2009; Heidenreich et al, 2011). Although extended pelvic LN dissection (ePLND) is the most accurate LN staging in patients undergoing RP for localised PCa (Abdollah et al, 2010; Heidenreich et al, 2011), PLND is not without morbidity and its therapeutical role is still a matter of debate (DiMarco et al, 2005; Briganti et al, 2006; Joslyn and Konety, 2006).

Previous tools have been developed to estimate the risk of LN metastasis and thereby guide the need and extent of PLND in PCa (Godoy et al, 2011; Briganti et al, 2012). It has been suggested that at least 10 LNs need to be removed and examined to achieve a 'standard' PLND (Touijer et al, 2007; Godoy et al, 2011), while at least 20 LNs removed and examined provides an ePLND (Weingartner et al, 1996). However, whether such numbers may vary according to individual patient characteristics is still unknown. This is key for planning the optimal treatment approach, given the strict correlation between PCa features and the risk of LN invasion (Heidenreich et al, 2002; Briganti et al, 2007; Abdollah et al, 2010).

We have recently developed a methodology that allows preoperative determination of the minimum number of LNs needed to take to ensure that a bladder cancer patient is correctly staged as LN negative (Shariat et al, 2012). The aim of this study was to develop a similar NSS for patients with PCa. We hypothesised that the true nodal status (no false-negative LN status) could be accurately predicted based on the number of LNs examined according to the clinical PCa features. Therefore, we used a large multicentre (i.e., heterogeneous) cohort of patients treated with RP and a variable extent of PLND to develop the novel NSS. Subsequently, we validated the novel model in a large single-centre cohort of RP patients who underwent an anatomically defined ePLND.

\section{PATIENTS AND METHODS}

Patients. The development cohort (DC) included data from 4770 patients treated with RP and PLND between 2000 and 2011 for PCa at eight high-volume centres. In this cohort, the extent of PLND was at the discretion of each treating physician, thus mainly consisting of an anatomically defined limited PLND, including removal of all lymphatic tissue in the obturator fossa and along the external iliac vessels. However, ePLND was performed too. The validation cohort (VC) included 3595 patients treated between 1989 and 2012 at a single centre with RP and anatomically defined ePLND for PCa. Here, the ePLND consisted of excision of fibrofatty tissue along the external iliac vein, with the distal limit being the deep circumflex vein and the femoral canal. Lymph nodes along the internal iliac artery were also removed. Proximally, ePLND included the bifurcation of the common iliac artery (Briganti et al 2012). Preoperative staging was performed with prostate-specific antigen (PSA), Gleason score at biopsy, digital rectal examination, and imaging study results. No patient received preoperative radiotherapy, hormonal treatment, or chemotherapy. No patient had clinically evident distant metastases at the time of RP. This was an institutional review board-approved study, with all participating sites providing the necessary institutional datasharing agreements prior to initiation of the study.

Pathological evaluation. Genitourinary pathologists assigned the pathological stage, which was reassigned according to the 2007 American Joint Committee on Cancer (AJCC) Tumor, Node, Metastasis (TNM) staging system when necessary. All lymphoid tissue removed was submitted for histological examination.

Statistical analysis. As in a previously described methodology for colorectal, bladder, and PCa (Gonen et al, 2009; Shariat et al, 2012; Kluth et al, 2013), we tested the probability of incorrect nodal staging as a function of the number of examined LNs. The true LN status is practically unascertainable as there is no way to ascertain the false-negative rate. However, information from LN-positive patients can be used to determine whether the number of LNs examined and the number of LNs that are negative are sufficient to classify a patient as LN negative. For example, one can consider a patient with a large number of examined LNs and small number of positive LNs (called $k$ ): if fewer LNs were examined, there would be a chance that this patient would be incorrectly deemed LN negative. In contrast, for a patient with a small number of examined LNs and large $k$, it is not likely that LN disease would have been missed, even though fewer LNs were examined. Thus, the data from LN-positive patients were used to interpret the data for the LN-negative patients. The probability that a LN-negative patient has LN disease can be computed using the following algorithm: computing the probability of missing a positive LN, computing the prevalence of pN1 status, and computing the NSS from sensitivity and prevalence.

Probability of missing a positive node. The probability of missing a positive LN (one minus the sensitivity) is inherent to the process of pathological detection and, as such, depends on the number of examined LNs - however, not on patient characteristics. For this purpose, we used a beta-binomial model, which allowed for heterogeneity in the intensity of LN spread across patients.

Three key assumptions underlie this step: (1) there are no false positives (if the specimen contains a negative $\mathrm{LN}$, it will be correctly identified by the pathologist); (2) all LNs are exchangeable; that is, they all have an equal probability of being involved; and (3) sensitivity is the same for LN-positive and LN-negative patients.

These assumptions may not be completely tenable, but we consider them to be sufficient approximations to our biological understanding of LN spread and clinical practice of nodal staging.

Estimation of prevalence of nodal disease. The observed prevalence is an underestimate and needed to be adjusted for false negatives, which was done in two steps. The first step comprises assumption 1 and estimates $\# \mathrm{FN}_{k}$ as a function of $k$, which is the number of positive LNs from patients with LN involvement:

$$
\# \mathrm{FN}_{k}=\frac{\left[1-P\left(\mathrm{FN}_{k}\right)\right] \times \# \mathrm{TP}_{k}}{P\left(\mathrm{FN}_{k}\right)}
$$

In this equation, $\# \mathrm{TP}_{k}$ is the number of true positives for a given $k$. Since prevalence is not a function of $k$, the second step receives the adjusted prevalence by averaging over $k$ :

$$
\text { Prev }=\frac{\sum_{k}\left(\mathrm{FN}_{k}+\mathrm{TP}_{k}\right)}{\sum_{k}\left(\mathrm{FN}_{k}+\mathrm{TP}_{k}+\mathrm{TN}_{k}\right)}
$$

Estimation of prevalence was stratified by clinical $\mathrm{T}$ stage, preoperative PSA, and Gleason score sum at biopsy for the cNSSs (preoperative). Furthermore, for a better clinical utilization of the cNSSs, prevalence was also estimated by a previously published clinical risk stratification (Briganti et al, 2006).

Nodal staging score. Adequate staging was assessed by computing the cNSS, the probability that a pathologically LN-negative patient is truly free from nodal disease:

$$
\mathrm{NSS}=\frac{1-\text { Prev }}{1-\text { Prev }+\left[\text { Prev } \times P\left(\mathrm{FN}_{k}\right)\right]}
$$

Confidence intervals. Precision of the reported estimates was assessed by creating 2000 bootstrap samples from the entire data set and replicating the estimation process. The 2.5th and 97.5th quartiles were used as the lower and upper 95\% confidence intervals for the corresponding estimates, respectively. 


\section{RESULTS}

Table 1 shows the clinical and pathological features of the 4770 patients in the DC and the 3595 patients in the VC. Among the 4770 patients in the DC, the median number of examined LNs was 6 (interquartile range (IQR): 7); $94.6 \%$ of the patients were deemed LN negative. Lymph node metastases were present in 16 of $3234(0.5 \%)$ pT2 patients, 85 of $1105(7.7 \%)$ pT3a patients, and 156 of $444(35.1 \%)$ pT3b/T4 patients. Among the 3595 patients in the VC, the median number of examined LNs was 16 (IQR: 11 ); $87.1 \%$ were free from LN metastasis. Lymph node metastases were present in 34 of 2381 (1.4\%) pT2 patients, 86 of 587 (14.7\%) pT3a patients, and 344 of 627 (54.9\%) pT3b/T4 patients.
In the DC, the resulting probability of missing nodal disease (one minus the sensitivity) as a function of the number of examined LNs is plotted in Figure 1A. As expected, the probability of missing LN disease decreased as the number of LNs examined increased: if only a single LN was examined in all patients, $84 \%$ of LN disease would be missed. The probability of missing a positive $\mathrm{LN}$ is $60 \%$ if $3 \mathrm{LNs}$ are examined, $45 \%$ if 5 are examined, and drops to $22 \%$ when 10 are examined. The sensitivity of nodal staging exceeds $80 \%$ only when at least $11 \mathrm{LNs}$ are examined. In the VC, as expected, the probability of missing LN metastasis decreases as the number of LNs examined increases (Figure 1B): if only a single LN is examined in all patients, $84 \%$ of LN disease would be missed. The probability of missing a positive LN decreases with an increasing number of LNs examined and is $62 \%$ if 3 LNs are examined, $47 \%$ if 5 are examined, and $30 \%$ if 10 are examined.

Table 1. Characteristics of 8365 patients who underwent radical prostatectomy and bilateral pelvic lymph node dissection for localised prostate cancer

Development cohort $(n=4770)$

Validation cohort $(n=3595)$

\begin{tabular}{|c|c|c|c|c|c|c|c|c|c|c|c|c|}
\hline & \multicolumn{2}{|c|}{ Patients } & \multicolumn{4}{|c|}{ Lymph nodes examined } & \multicolumn{2}{|c|}{ Patients } & \multicolumn{4}{|c|}{ Lymph nodes examined } \\
\hline & No. & $\%$ & Mean & s.d. & Median & IQR & No. & $\%$ & Mean & s.d. & Median & IQR \\
\hline \multicolumn{13}{|c|}{ Clinical T stage } \\
\hline cT1 & 3298 & 69.1 & 6.9 & 6.0 & 5.0 & 6 & 1883 & 52.4 & 17.2 & 8.1 & 16.0 & 11 \\
\hline cT2 & 1367 & 28.7 & 8.2 & 6.5 & 6.0 & 8 & 1231 & 34.2 & 17.5 & 8.4 & 16.0 & 11 \\
\hline cT3 & 105 & 2.2 & 9.2 & 6.2 & 7.0 & 7 & 481 & 13.4 & 19.5 & 10.0 & 18.0 & 12 \\
\hline
\end{tabular}

\section{Bx Gleason score}

\begin{tabular}{|c|c|c|c|c|c|c|c|c|c|c|c|c|}
\hline 6 & 2121 & 44.5 & 5.94 & 5.0 & 5.0 & 5 & 2230 & 62.0 & 17.1 & 7.9 & 16.0 & 11 \\
\hline 7 & 2063 & 43.2 & 8.0 & 6.6 & 6.0 & 8 & 1020 & 28.4 & 17.9 & 8.6 & 17.0 & 11 \\
\hline $8-10$ & 586 & 12.3 & 9.7 & 7.6 & 9.0 & 8 & 345 & 9.6 & 20.1 & 11.1 & 18.0 & 13 \\
\hline
\end{tabular}

Pathological T stage

\begin{tabular}{|c|c|c|c|c|c|c|c|c|c|c|c|c|}
\hline pT2 & 3234 & 67.6 & 6.8 & 5.7 & 5.0 & 6 & 2381 & 66.2 & 17.9 & 8.0 & 16.0 & 11 \\
\hline pT3a & 1103 & 23.1 & 8.2 & 6.6 & 6.0 & 7 & 587 & 16.3 & 18.2 & 8.6 & 17.0 & 11 \\
\hline pT3b/T4 & 443 & 9.3 & 9.7 & 7.4 & 8.0 & 9 & 627 & 17.4 & 19.1 & 9.9 & 17.0 & 10 \\
\hline
\end{tabular}

RP Gleason score

\begin{tabular}{|c|c|c|c|c|c|c|c|c|c|c|c|c|}
\hline 6 & 1135 & 23.8 & 5.6 & 4.4 & 5.0 & 4 & 1387 & 38.6 & 17.0 & 7.9 & 16.0 & 11 \\
\hline 7 & 3170 & 66.4 & 7.7 & 6.4 & 6.0 & 7 & 1685 & 46.9 & 17.4 & 8.3 & 16.0 & 11 \\
\hline $8-10$ & 467 & 9.8 & 9.1 & 7.3 & 7.0 & 9 & 523 & 14.5 & 20.0 & 10.1 & 18.0 & 11 \\
\hline
\end{tabular}

Surgical margin

\begin{tabular}{|c|c|c|c|c|c|c|c|c|c|c|c|c|}
\hline $\begin{array}{l}\text { Negative } \\
\text { Positive }\end{array}$ & $\begin{array}{r}4029 \\
741\end{array}$ & $\begin{array}{l}84.5 \\
15.5\end{array}$ & $\begin{array}{l}7.2 \\
8.3\end{array}$ & $\begin{array}{l}6.1 \\
6.5\end{array}$ & $\begin{array}{l}5.0 \\
6.0\end{array}$ & $\begin{array}{l}6 \\
8\end{array}$ & $\begin{array}{l}2588 \\
1007\end{array}$ & $\begin{array}{l}72.0 \\
28.0\end{array}$ & $\begin{array}{l}17.2 \\
18.7\end{array}$ & $\begin{array}{l}8.1 \\
9.3\end{array}$ & $\begin{array}{l}16.0 \\
17.0\end{array}$ & $\begin{array}{l}11 \\
11\end{array}$ \\
\hline \multicolumn{13}{|c|}{ Pathological N stage } \\
\hline $\begin{array}{l}\mathrm{NO} \\
\mathrm{N}+\end{array}$ & $\begin{array}{r}4514 \\
256\end{array}$ & $\begin{array}{r}94.6 \\
5.4\end{array}$ & $\begin{array}{r}7.2 \\
10.4\end{array}$ & $\begin{array}{l}6.0 \\
8.1\end{array}$ & $\begin{array}{r}6.0 \\
10.0\end{array}$ & $\begin{array}{l}6 \\
6\end{array}$ & $\begin{array}{r}3131 \\
464\end{array}$ & $\begin{array}{l}87.1 \\
12.9\end{array}$ & $\begin{array}{l}17.1 \\
21.3\end{array}$ & $\begin{array}{r}7.9 \\
11.0\end{array}$ & $\begin{array}{l}16.0 \\
19.0\end{array}$ & $\begin{array}{l}11 \\
11\end{array}$ \\
\hline
\end{tabular}

\begin{tabular}{l} 
Preoperative PSA (ng ml ${ }^{-1}$ ) \\
\hline$<10$ \\
\hline 10
\end{tabular}

\begin{tabular}{|c|c|c|c|c|c|c|c|c|}
\hline & \multicolumn{4}{|c|}{ Development cohort $(n=4770)$} & \multicolumn{4}{|c|}{ Validation cohort $(n=3595)$} \\
\hline & Median & Mean & Range & IQR & Median & Mean & Range & IQR \\
\hline Age (years) & 62.0 & 61.3 & $45-82$ & 10 & 66.0 & 65.5 & $40-89$ & 10 \\
\hline No. of examined LNs in all patients & 6.0 & 7.4 & $1-55$ & 7 & 16.0 & 17.6 & $5-73$ & 11 \\
\hline No. of examined LNs in patients with negetive LNs & 6.0 & 7.2 & $1-55$ & 6 & 16.0 & 17.8 & $5-66$ & 11 \\
\hline No. of examined LNs in patients with positive LNs & 10.0 & 10.4 & $1-38$ & 10 & 19.0 & 21.3 & $5-73$ & 11 \\
\hline No. of positive LNs in patients with positive LNs & 1.0 & 1.8 & $1-23$ & 1 & 2.0 & 3.81 & 1.6 & 2 \\
\hline
\end{tabular}


Sensitivity of nodal staging exceeds $80 \%$ only when at least 15 LNs are examined.

The results for cNSS in the DC are shown in Figure 2 and Table 2. Although patients with a cT1 tumour have a $90 \%$ probability to be free of LN metastasis with one single LN examined, patients with cT2 and cT3 tumours will need 6 and 11 LNs examined to maintain similar levels of cNSS, respectively (Figure 2A). Although patients in the low- and intermediate-risk group achieve a cNSS of $90 \%$ with any examined LN, patients in the high-risk group will require eight examined LNs to achieve a similar probability (Figure 2D).

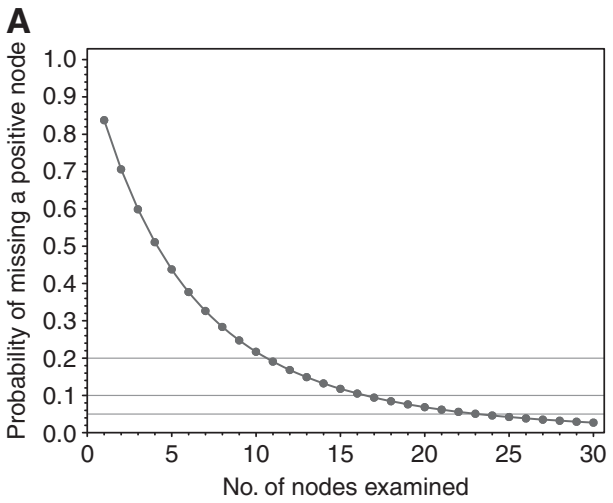

Figure 3 and Table 3 show the results for cNSS in the VC. For cT1 tumours, six examined LNs provide $90 \%$ confidence that the patient was indeed LN negative. For the same level of confidence, one would need 8 for cT2 tumours. Patients with a cT3 tumour will reach a cNSS of only $80 \%$ with 11 LNs examined, and $90 \%$ with 20 LNs examined (Figure 3A). Similar to the DC, in patients in the low- and intermediate-risk group, any LNs examined will result in a cNSS of $>90 \%$, whereas patients in the high-risk group will need 10 LNs examined to achieve a probability of $80 \%$ to be truly LN negative (Figure 3D).

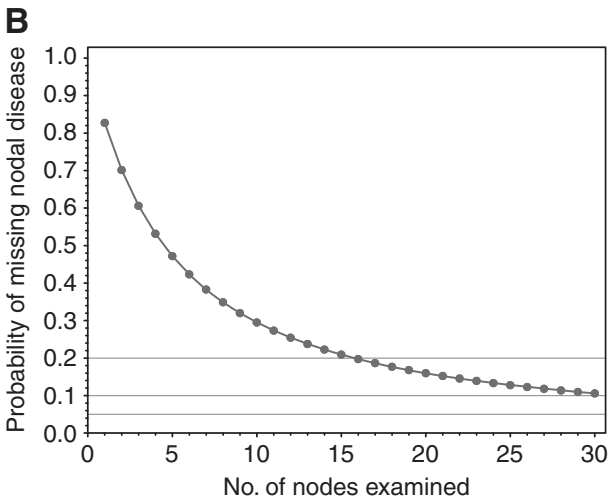

Figure 1. (A) Probability of missing nodal disease as a function of nodes examined in the development cohort of 4770 patients who were treated with radical prostatectomy and bilateral pelvic lymphadenectomy for localised prostate cancer. (B) Probability of missing nodal disease as a function of nodes examined in the validation cohort of 3595 patients who were treated with radical prostatectomy and bilateral pelvic lymphadenectomy for localised prostate cancer.

A

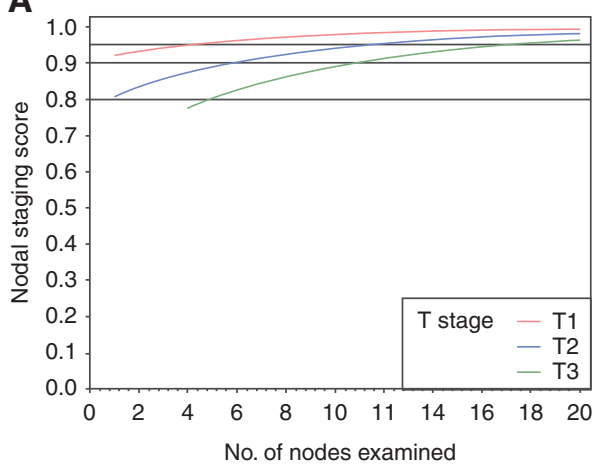

C

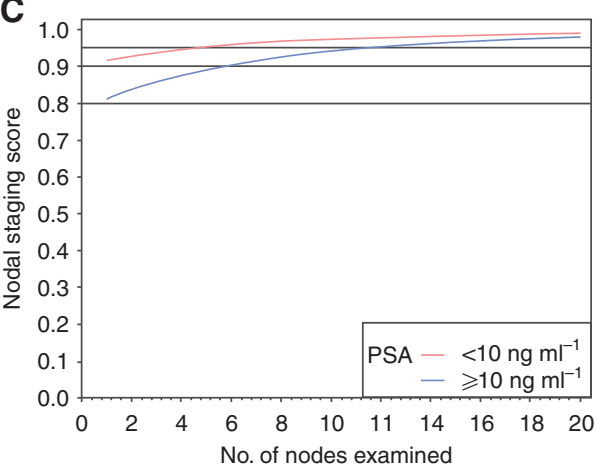

B

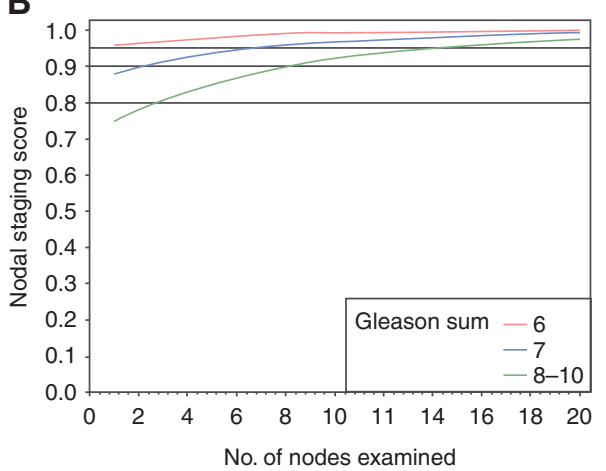

D

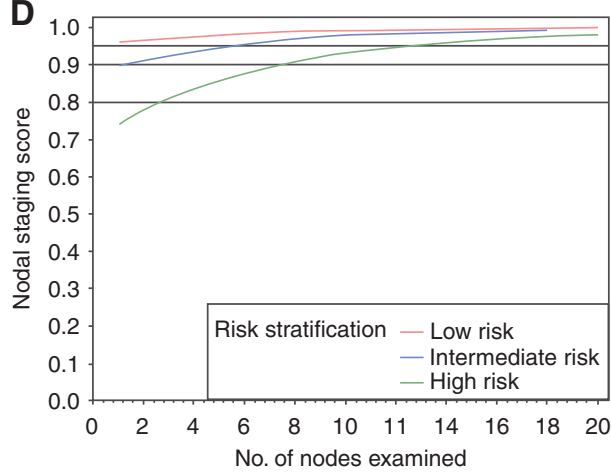

Figure 2. Clinical nodal staging scores in the development cohort of 4770 patients who were treated with radical prostatectomy and bilateral pelvic lymphadenectomy for localised prostate cancer. The vertical axis is the probability of missing nodal disease (one minus sensitivity); the horizontal axis is the number of examined nodes. (A) Sensitivity of the pathological evaluation of nodal disease stratified by clinical tumour stage. (B) Sensitivity of the pathological evaluation of nodal disease stratified by biopsy Gleason score. (C) Sensitivity of the pathological evaluation of nodal disease stratified by preoperative prostate-specific antigen. (D) Sensitivity of the pathological evaluation of nodal disease stratified by preoperative risk grouping. 
Table 2. Clinical nodal staging scores for selected values of the number of $L$ Ns examined in the development cohort of 4770 patients who underwent radical prostatectomy and bilateral pelvic lymph node dissection for localised prostate cancer

\begin{tabular}{|l|c|c|c|c|c|c|c|c|}
\hline $\begin{array}{l}\text { No. of } \\
\text { examined LNs }\end{array}$ & $\mathbf{1}$ & $\mathbf{3}$ & $\mathbf{5}$ & $\mathbf{8}$ & $\mathbf{1 0}$ & $\mathbf{1 2}$ & $\mathbf{1 5}$ & $\mathbf{2 0}$ \\
\hline \multicolumn{7}{|l|}{ Clinical T stage } \\
\hline T1 & 92.4 & 94.4 & 95.9 & 97.3 & 97.9 & 98.4 & 98.9 & 99.3 \\
T2 & 80.7 & 85.4 & 88.9 & 92.5 & 94.2 & 96.4 & 96.8 & 98.1 \\
T3 & - & - & 80.3 & 86.3 & 89.2 & 91.4 & 93.8 & 96.3 \\
\hline Bx Gleason score \\
\hline 6 & 95.5 & 96.8 & 97.6 & 98.5 & 98.8 & 99.0 & 99.4 & 99.6 \\
7 & 88.0 & 91.2 & 93.4 & 95.6 & 96.6 & 97.4 & 98.1 & 98.9 \\
$8-10$ & 74.8 & 80.6 & 85.0 & 89.8 & 92.0 & 93.7 & 95.5 & 97.3 \\
\hline
\end{tabular}

Preoperative PSA $\left(\mathrm{ng} \mathrm{ml}^{-1}\right)$

\begin{tabular}{|l|l|l|l|l|l|l|l|l|}
$<10$ & 91.9 & 94.1 & 95.6 & 97.1 & 97.8 & 98.3 & 98.8 & 99.3 \\
& 81.5 & 86.1 & 89.4 & 92.9 & 94.5 & 95.7 & 96.9 & 98.2 \\
\hline
\end{tabular}

\begin{tabular}{l|l|l|l|l|l|l|l|l|}
$\geqslant 10$ & 81.5 & 86.1 & 89.4 & 92.9 & 94.5 & 95.7 & 96.9 & 98.2 \\
\hline
\end{tabular}

Risk stratification

\begin{tabular}{|l|l|l|l|l|l|l|l|l|}
\hline Low risk & 96.1 & 97.3 & 98.1 & 98.8 & 99.1 & 99.4 & 99.6 & 99.8 \\
Intermediate risk & 89.6 & 92.6 & 94.7 & 96.7 & 97.6 & 98.2 & 98.8 & 99.2 \\
High risk & 74.3 & 80.8 & 85.7 & 90.8 & 93.1 & 94.7 & 96.5 & 98.1 \\
\hline
\end{tabular}

Abbreviations: $\mathrm{LNs}=$ lymph nodes; PSA = prostate-specific antigen.

Table 3. Clinical nodal staging scores for selected values of the number of LNs examined in the validation cohort of 3595 patients who underwent radical prostatectomy and bilateral pelvic lymph node dissection for localised prostate cancer

\begin{tabular}{|l|c|c|c|c|c|c|c|c|}
\hline $\begin{array}{l}\text { No. of examined } \\
\text { LNs }\end{array}$ & $\mathbf{1}$ & $\mathbf{3}$ & $\mathbf{5}$ & $\mathbf{8}$ & $\mathbf{1 0}$ & $\mathbf{1 2}$ & $\mathbf{1 5}$ & $\mathbf{2 0}$ \\
\hline Clinical T stage \\
\hline T1 & - & - & 94.2 & 95.1 & 95.8 & 96.9 & 97.3 & 97.7 \\
T2 & - & - & - & 90.0 & 91.9 & 92.6 & 94.3 & 95.2 \\
T3 & - & - & - & 75.1 & 78.8 & 80.9 & 84.9 & 88.3 \\
\hline
\end{tabular}

\section{Bx Gleason score}

\begin{tabular}{|l|c|c|c|c|c|c|c|c|}
\hline 6 & - & - & 95.0 & 95.8 & 97.2 & 97.5 & 97.7 & 98.2 \\
7 & - & - & 84.0 & 87.5 & 89.1 & 90.2 & 92.5 & 94.6 \\
$8-10$ & - & - & - & 67.1 & 70.0 & 71.9 & 76.6 & 81.9 \\
\hline
\end{tabular}

Preoperative PSA $\left(\mathrm{ng} \mathrm{ml}^{-1}\right)$

\begin{tabular}{|l|l|l|l|l|l|l|l|l|}
\hline$<10$ & - & - & 93.6 & 95.2 & 95.9 & 96.4 & 97.0 & 97.7 \\
$\geqslant 10$ & - & - & 78.0 & 82.7 & 85.0 & 86.8 & 88.9 & 91.3 \\
\hline
\end{tabular}

Risk stratification

\begin{tabular}{l|c|c|c|c|c|c|c|c|} 
Low risk & - & - & - & 95.6 & 96.2 & 96.7 & 97.1 & 97.9 \\
Intermediate risk & - & - & 92.3 & 94.2 & 95.0 & 95.7 & 96.4 & 97.3 \\
High risk & - & - & 70.3 & 76.2 & 79.1 & 81.5 & 84.2 & 87.5
\end{tabular}

Abbreviations: $L N s=$ lymph nodes; PSA = prostate-specific antigen.

\section{DISCUSSION}

In patients undergoing RP for PCa, PLND is an essential procedure for accurate LN staging (Abdollah et al, 2010; Heidenreich et al, 2011). Lymph node metastasis is a powerful prognostic factor in these patients (Briganti et al, 2009; Heidenreich et al, 2011) guiding clinical decision-making regarding patient counselling, follow-up scheduling and adjuvant therapies (Steuber et al, 2006; Boorjian et al, 2007; Abdollah et al, 2012). Many efforts have been made to estimate the number of LNs needed to be removed and examined in order to determine the true LN status (Heidenreich et al, 2002; Bader et al, 2003; Briganti et al, 2007; Abdollah et al, 2012). Anywhere between 10 and $28 \mathrm{LNs}$ removed and examined has been recommended for accurate LN staging (Briganti et al, 2007; Touijer et al, 2007). The discrepancy between studies in the number of LNs needed to be removed may be due to the extent of dissection, pathological factors, variability in patients' pelvic anatomy, and variability of disease severity (Bochner et al, 2001; Mattei et al, 2008; Joniau et al, 2013). Moreover, all these studies share one significant limitation: the lack of adjustment for the effect of clinical factors. This is crucial, given the strict association between cancer aggressiveness and rate of LN metastasis (Carlsson et al, 2013). Therefore, in this study we aimed to assess the association between the number of examined LNs and the true LN status according to preoperative patient characteristics.

We found that the number of LNs needed to be removed varies largely among patients according to clinical PCa features. The proportion of having a positive LN increased indeed proportionally with advancing clinical $\mathrm{T}$ stage, higher biopsy Gleason score, and higher PSA. Moreover, we found that all preoperative characteristics are highly correlated with the number of LNs needed to be examined to ascertain the true LN status. One might argue that such associations, where more aggressive cancers need more extensive PLND to detect a single nodal metastasis, seem to be counter-intuitive. In fact, while only few LNs are needed to be removed, ascertaining that a low-risk patient is LN negative is mainly driven by the extremely low rate of LNI in this patient group. Indeed, despite extension of the PLND to all anatomical nodal pelvic areas, the rate of LNI in this patient group remains $<2 \%$ (Weingartner et al, 1996). Conversely, the notion that more LNs are required to achieve accurate LN-negative staging in men at higher risk of LNI is mainly due to the highly heterogenous and complex anatomical pathway of LN metastasis in PCa (Shariat et al, 2003a,b). Nodal dissemination in PCa does not indeed follow a pre-defined pathway of metastatic spread, but rather different lymphatic routes tributary to several primary lymphatic landing sites (Mattei et al, 2008). However, one has to realise that even with extensive PLND a $90 \%$ probability to be free from nodal metastasis will not be achieved. Therefore, even a well-performed, meticulous ePLND does not ensure $100 \%$ accuracy with regard to nodal status. Moreover, the risk of nodal invasion is not driven by a single cancer characteristic, but is highly dependent on the interaction of several clinical variables.

Unfortunately, the extent of PLND is too often based on the surgeon's intuitive experience integrating his/her beliefs, patient characteristics such as performance status, comorbidities and tumour characteristics. Our model proves the concept that the needed extent of PLND is dependent on and can be predicted by the risk of LNI based on individual patient features, similar to a model we previously described for PCa (Kluth et al, 2013). Indeed, the one-size-fits-all approach is too inaccurate and one needs to estimate the individual risk of missing a positive LN. Performing an ePLND in all patients would certainly result in overtreatment of many patients, with resulting side effects and cost. Conversely, removing too few LNs may result in inaccurate staging and possibly inferior survival (Bader et al, 2003; Joslyn and Konety, 2006). Our tool may enable the physician and the patient to join in the decision-making by identifying risks through weighting the probability of missing a positive LN taking into account the risk of PLND. 
A

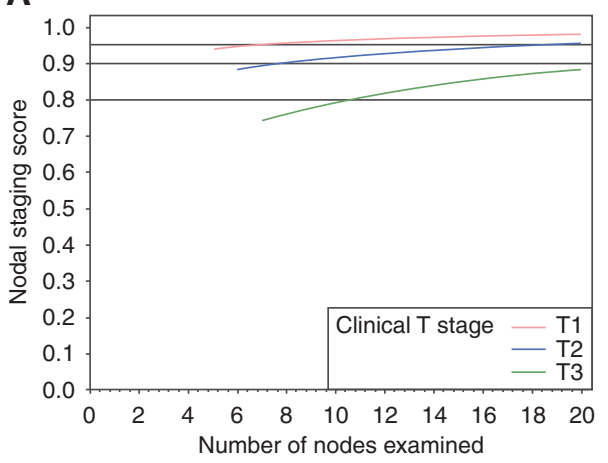

C

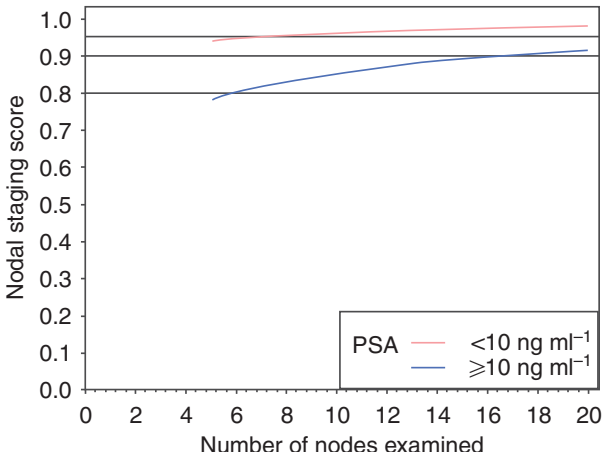

B

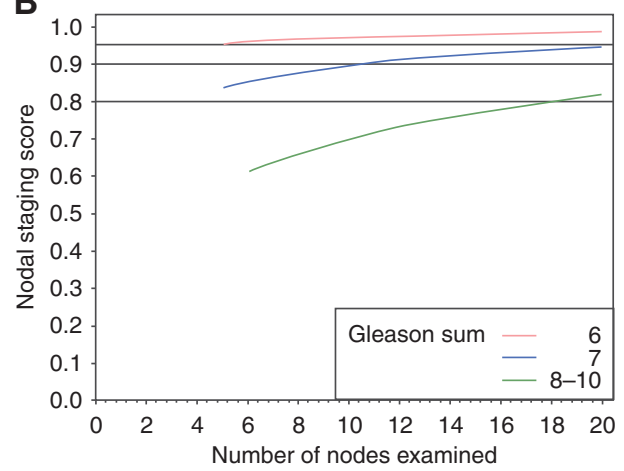

D

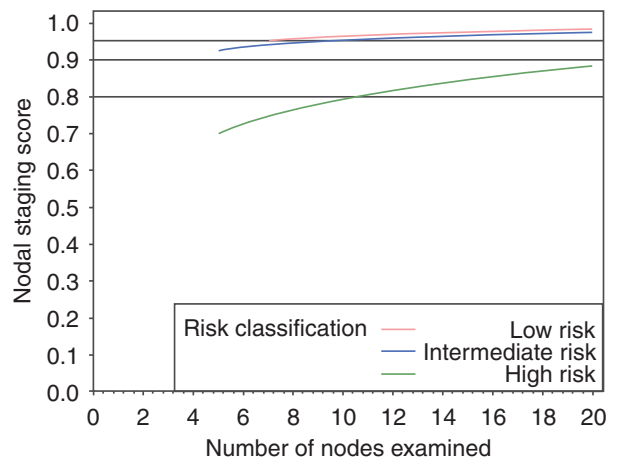

Figure 3. Clinical nodal staging scores in the validation cohort of 3595 patients who were treated with radical prostatectomy and bilateral pelvic lymphadenectomy for localised prostate cancer. The vertical axis is the probability of missing nodal disease (one minus sensitivity); the horizontal axis is the number of examined nodes. (A) Sensitivity of the pathological evaluation of nodal disease stratified by clinical tumour stage.

(B) Sensitivity of the pathological evaluation of nodal disease stratified by biopsy Gleason score. (C) Sensitivity of the pathological evaluation of nodal disease stratified by preoperative prostate-specific antigen. (D) Sensitivity of the pathological evaluation of nodal disease stratified by preoperative risk grouping.

Although we were able to control for numerous potential confounders, we could not control for the surgeon's and pathologist's experience or treatment decisions. In the DC, we could not adjust for the anatomic template of the preferred PLND and the location of each removed LN, which has been shown to be important (Weingartner et al, 1996). Removing LNs from an area of high likelihood of malignancy may be more valuable than removing a LN that is less likely to be involved with cancer (Joniau and Van den Bergh, 2013). However, this limitation was not applicable to the VC, where all patients underwent an anatomically defined ePLND regardless of the risk of LNI. Nevertheless, one might argue that the number of LNs examined is not an exact surrogate for the extent or template of PLND. In addition, the number of LNs examined is also dependent on pathological evaluation and inherent differences among patients (Bochner et al, 2004). Specific characteristics of the DC and VC may increase the generalisability of this tool as data were derived from two different patient cohorts: a DC, in which the extent of PLND was based on the surgeon's discretion, and a VC, which was based on a large single-centre experience with a protocol for ePLND. Thus, our study examined the differential impact of the extent of PLND; it is noteworthy that the probability of freedom from missed LN metastasis was similar in both cohorts, thus supporting its generalisability and thereby proving its robustness. Finally, our model is based on assumptions. Although these assumptions might seem debatable, every single mathematical model and every theory is built on assumptions tested in an experimental setting, such as that used in this study. Prospective validation of our model can test whether the assumptions were realistic and robust (Kattan, 2011).

\section{CONCLUSIONS}

For patients treated with RP for PCa, PLND is the only reliable technique to ensure accurate nodal staging. The minimum number of examined LN staging may be predictable, being strongly dependent on preoperative PCa characteristics. We developed a model to allow an informed decision-making based on these characteristics.

\section{REFERENCES}

Abdollah F, Sun M, Thuret R, Budaus L, Jeldres C, Graefen M, Briganti A, Perrotte P, Rigatti P, Montorsi F, Karakiewicz PI (2010) Decreasing rate and extent of lymph node staging in patients undergoing radical prostatectomy may undermine the rate of diagnosis of lymph node metastases in prostate cancer. Eur Urol 58(6): $882-892$.

Abdollah F, Suardi N, Cozzarini C, Gallina A, Capitanio U, Bianchi M, Sun M, Fossati N, Passoni NM, Fiorino C, Di Muzio N, Karakiewicz PI, Rigatti P, Montorsi F, Briganti A (2012) Selecting the optimal candidate for adjuvant radiotherapy after radical prostatectomy for prostate cancer: a long-term survival analysis. Eur Urol 63(6): 998-1008.

Abdollah F, Sun M, Thuret R, Jeldres C, Tian Z, Briganti A, Shariat SF, Perrotte P, Montorsi F, Karakiewicz PI (2012) Lymph node count threshold for optimal pelvic lymph node staging in prostate cancer. Int J Urol 19(7): 645-651.

Briganti A, Blute ML, Eastham JH, Graefen M, Heidenreich A, Karnes JR, Montorsi F, Studer UE (2009) Pelvic lymph node dissection in prostate cancer. Eur Urol 55(6): 1251-1265. 
Briganti A, Chun FK, Salonia A, Suardi N, Gallina A, Da Pozzo LF, Roscigno M, Zanni G, Valiquette L, Rigatti P, Montorsi F, Karakiewicz PI (2006) Complications and other surgical outcomes associated with extended pelvic lymphadenectomy in men with localized prostate cancer. Eur Urol 50(5): 1006-1013.

Briganti A, Larcher A, Abdollah F, Capitanio U, Gallina A, Suardi N, Bianchi M, Sun M, Freschi M, Salonia A, Karakiewicz PI, Rigatti P, Montorsi F (2012) Updated nomogram predicting lymph node invasion in patients with prostate cancer undergoing extended pelvic lymph node dissection: the essential importance of percentage of positive cores. Eur Urol 61(3): 480-487.

Briganti A, Chun FK, Salonia A, Gallina A, Zanni G, Scattoni V, Valiquette L, Rigatti P, Montorsi F, Karakiewicz PI (2007) Critical assessment of ideal nodal yield at pelvic lymphadenectomy to accurately diagnose prostate cancer nodal metastasis in patients undergoing radical retropubic prostatectomy. Urology 69(1): 147-151.

Briganti A, Chun FK, Salonia A, Gallina A, Farina E, Da Pozzo LF, Rigatti P, Montorsi F, Karakiewicz PI (2006) Validation of a nomogram predicting the probability of lymph node invasion based on the extent of pelvic lymphadenectomy in patients with clinically localized prostate cancer. BJU Int 98(4): 788-793.

Boorjian SA, Thompson RH, Siddiqui S, Bagniewski S, Bergstralh EJ, Karnes RJ, Frank I, Blute ML (2007) Long-term outcome after radical prostatectomy for patients with lymph node positive prostate cancer in the prostate specific antigen era. J Urol 178(3 Pt 1): 864-870; discussion $70-1$.

Bader P, Burkhard FC, Markwalder R, Studer UE (2003) Disease progression and survival of patients with positive lymph nodes after radical prostatectomy. Is there a chance of cure? J Urology 169(3): 849-854.

Bochner BH, Herr HW, Reuter VE (2001) Impact of separate versus en bloc pelvic lymph node dissection on the number of lymph nodes retrieved in cystectomy specimens. J Urol 166(6): 2295-2296.

Bochner BH, Cho D, Herr HW, Donat M, Kattan MW, Dalbagni G (2004) Prospectively packaged lymph node dissections with radical cystectomy: evaluation of node count variability and node mapping. J Urol 172(4 Pt 1): 1286-1290.

Carlsson SV, Tafe LJ, Chade DC, Sjoberg DD, Passoni N, Shariat SF, Eastham J, Scardino PT, Fine SW, Touijer KA (2013) Pathological features of lymph node metastasis for predicting biochemical recurrence after radical prostatectomy for prostate cancer. J Urol 189(4): 1314-1319.

DiMarco DS, Zincke H, Sebo TJ, Slezak J, Bergstralh EJ, Blute ML (2005) The extent of lymphadenectomy for pTXNO prostate cancer does not affect prostate cancer outcome in the prostate specific antigen era. J Urol 173(4): $1121-1125$.

Godoy G, Chong KT, Cronin A, Vickers A, Laudone V, Touijer K, Guillonneau B, Eastham JA, Scardino PT, Coleman JA (2011) Extent of pelvic lymph node dissection and the impact of standard template dissection on nomogram prediction of lymph node involvement. Eur Urol 60(2): 195-201.

Gonen M, Schrag D, Weiser MR (2009) Nodal staging score: a tool to assess adequate staging of node-negative colon cancer. J Clin Oncol 27(36): 6166-6171.

Heidenreich A, Bellmunt J, Bolla M, Joniau S, Mason M, Matveev V, Mottet N, Schmid HP, van der Kwast T, Wiegel T, Zattoni F. European Association of Urology (2011) EAU guidelines on prostate cancer. Part 1: screening, diagnosis, and treatment of clinically localised disease. Eur Urol 59(1): 61-71.

Heidenreich A, Varga Z, Von Knobloch R (2002) Extended pelvic lymphadenectomy in patients undergoing radical prostatectomy: high incidence of lymph node metastasis. J Urol 167(4): 1681-1686.

Joslyn SA, Konety BR (2006) Impact of extent of lymphadenectomy on survival after radical prostatectomy for prostate cancer. Urology 68(1): 121-125.
Joniau S, Van den Bergh L, Lerut E, Deroose CM, Haustermans K, Oyen R, Budiharto T, Ameye F, Bogaerts K, Van Poppel H (2013) Mapping of pelvic lymph node metastases in prostate cancer. Eur Urol 63(3): 450-458.

Joniau S, Van den Bergh L (2013) Reply to Henk G. van der Poel, Tessa Buckle and Renato Valdes Olmos' Letter to the Editor re: Steven Joniau, Laura Van den Bergh, Evelyne Lerut, et al. Mapping of Pelvic Lymph Node Metastases in Prostate Cancer. Eur Urol. In press. http://dx.doi.org/ 10.1016/j.eururo.2012.06.057. Eur Urol 63(2): e21-e22.

Kluth LA, Abdollah F, Xylinas E, Rieken M, Fajkovic H, Sun M, Karakiewicz PI, Seitz C, Schramek P, Herman MP, Becker A, Loidl W, Pummer K, Nonis A, Lee RK, Lotan Y, Scherr DS, Seiler D, Chun FK, Graefen M, Tewari A, Gönen M, Montorsi F, Shariat SF, Briganti A (2013) Pathologic nodal staging scores in patients treated with radical prostatectomy: a postoperative decision tool. Eur Urol S0302-2838(13): 00656-00658.

Kattan MW (2011) Factors affecting the accuracy of prediction models limit the comparison of rival prediction models when applied to separate data sets. Eur Urol 59(4): 566-567.

Mattei A, Fuechsel FG, Bhatta Dhar N, Warncke SH, Thalmann GN, Krause T, Studer UE (2008) The template of the primary lymphatic landing sites of the prostate should be revisited: results of a multimodality mapping study. Eur Urol 53(1): 118-125.

Shariat SF, Ehdaie B, Rink M, Cha EK, Svatek RS, Chromecki TF, Fajkovic H, Novara G, David SG, Daneshmand S, Fradet Y, Lotan Y, Sagalowsky AI, Clozel T, Bastian PJ, Kassouf W, Fritsche HM, Burger M, Izawa JI, Tilki D, Abdollah F, Chun FK, Sonpavde G, Karakiewicz PI, Scherr DS, Gonen M (2012) Clinical nodal staging scores for bladder cancer: a proposal for preoperative risk assessment. Eur Urol 61(2): 237-242.

Shariat SF, Rink M, Ehdaie B, Xylinas E, Babjuk M, Merseburger AS, Svatek RS, Cha EK, Tagawa ST, Fajkovic H, Novara G, Karakiewicz PI, Trinh QD, Daneshmand S, Lotan Y, Kassouf W, Fritsche HM, Chun FK, Sonpavde G, Joual A, Scherr DS, Gonen M (2012) Pathologic nodal staging score for bladder cancer: a decision tool for adjuvant therapy after radical cystectomy. Eur Urol 63(2): 371-378.

Steuber T, Budaus L, Walz J, Zorn KC, Schlomm T, Chun F, Ahyai S, Fisch M, Sauter G, Huland H, Graefen M, Haese A (2006) Radical prostatectomy improves progression-free and cancer-specific survival in men with lymph node positive prostate cancer in the prostate-specific antigen era: a confirmatory study. BJU Int 107(11): 1755-1761.

Shariat SF, Roudier MP, Wilcox GE, Kattan MW, Scardino PT, Vessella RL, Erdamar S, Nguyen C, Wheeler TM, Slawin KM (2003a) Comparison of immunohistochemistry with reverse transcription-PCR for the detection of micrometastatic prostate cancer in lymph nodes. Cancer Res 63(15): 4662-4670.

Shariat SF, Kattan MW, Erdamar S, Nguyen C, Scardino PT, Spencer DM, Wheeler TM, Slawin KM (2003b) Detection of clinically significant, occult prostate cancer metastases in lymph nodes using a splice variantspecific rt-PCR assay for human glandular kallikrein. J Clin Oncol 21(7): $1223-1231$.

Touijer K, Rabbani F, Otero JR, Secin FP, Eastham JA, Scardino PT, Guillonneau B (2007) Standard versus limited pelvic lymph node dissection for prostate cancer in patients with a predicted probability of nodal metastasis greater than 1\%. J Urol 178(1): 120-124.

Weingartner K, Ramaswamy A, Bittinger A, Gerharz EW, Voge D, Riedmiller H (1996) Anatomical basis for pelvic lymphadenectomy in prostate cancer: results of an autopsy study and implications for the clinic. J Urol 156(6): 1969-1971.

This work is published under the standard license to publish agreement. After 12 months the work will become freely available and the license terms will switch to a Creative Commons AttributionNonCommercial-Share Alike 3.0 Unported License. 